\title{
SAXS-Fiber Computer Tomography. Method Enhancement and Analysis of Microfibrillar-Reinforced Composite Precursors from PEBA and PET
}

\author{
Norbert Stribeck, ${ }^{*},{ }^{\dagger}$ Ulrich Nöchel, ${ }^{\dagger}$ Stoyko Fakirov, ${ }^{\ddagger}$ Jan Feldkamp, ${ }^{\S}$ \\ Christian Schroer, ${ }^{\S}$ Andreas Timmann, ${ }^{\prime \prime}$ and Marion Kuhlmann"
}

\begin{abstract}
Department of Chemistry, Institute of Technical and Macromolecular Chemistry, University of Hamburg, Bundesstrasse 45, 20146 Hamburg, Germany; Mechanical Engineering Department, University of Auckland, Private Bag 92019, Auckland, New Zealand; Institute of Structure Physics, Technical University of Dresden, 01062 Dresden, Germany; and HASYLAB at DESY, Notkestrasse 85, 22603 Hamburg, Germany
\end{abstract}

Received July 8, 2008; Revised Manuscript Received August 11, 2008

\begin{abstract}
The nanostructure gradient along the fiber radius inside polymer strands is uncovered by smallangle X-ray scattering (SAXS) microbeam-scanning experiments and X-ray scattering fiber computer-tomography (XSF-CT) analysis. We notice restricted visibility of scattering features within the series of patterns. The reason is violation of local fiber symmetry (LFS) in the irradiated volume elements (voxels). For its theoretical treatment, a set of elementary topologies (tangential grain, radial grain) is introduced. Systematic aberrations (ultrareconstruction, infrareconstruction) generated by tomographic reconstruction of affected series are described. A concept for handling and utilization of these aberrations for nanostructure analysis is devised. Precursors of polymer microfibrillar-reinforced composites (MFC) containing poly(ether)-block-amide (PEBA) and poly(ethylene terephthalate) (PET) with varying cold-draw ratio are studied. We compare results from a direct analysis of the smeared measured patterns to results obtained after tomographic reconstruction and fathom the power of reconstruction methods. Ideas for advanced practical applications of the XSF-CT method are discussed.
\end{abstract}

\section{Introduction}

In polymer engineering, parts with cylindrical symmetry are frequently made. These are not only fibers but also extruded strands and pipes that often are postprocessed by exposure to mechanical and thermal load. Based on coextrudates from various polymers and nanoparticles, great efforts are made to control the nanostructure by smart processing, aiming at materials with tailored properties. One class of such materials are the microfibrillar-reinforced composites ${ }^{1-5}$ (MFC). MFC are blends from two immiscible polymers with controlled morphology. One component forms the matrix, in which reinforcing fibrils of the other component are distributed. Their manufacturing includes three processing steps: (1) melt blending of the starting neat polymers and extrusion, (2) cold drawing of the blend, and (3) subsequent annealing of the drawn blend at constant strain and at $T_{\mathrm{m} 1}<T<T_{\mathrm{m} 2}$, where $T_{\mathrm{m} 1}$ is the melting temperature of the lower melting component and $T_{\mathrm{m} 2}$ is that of the higher melting one. With controlled processing, the diameter of the fibrils and their length and alignment as well as their adhesion to the matrix can be controlled. In such a way, different composites can be produced from the same polymer blend aiming at improvement of the tensile and flexural properties of the matrix material.

Spatially resolved X-ray scattering studies of fibers and strands are quite common, because the data from a single scan is almost all one can get for an investigation of the radial structure gradient. ${ }^{6-20}$ In this process, the fiber is irradiated in the direction perpendicular to the fiber axis by a fine X-ray beam that is stepwise translated across the fiber. The result is a series of projected scattering patterns, which vary as a function of the

\footnotetext{
* Corresponding author.

University of Hamburg.

$\div$ University of Auckland.

$\S$ Technical University of Dresden.

"HASYLAB at DESY.
}

scan position $\mathrm{x}$ on the fiber diameter. In general, this variation is interpreted and qualitatively related to structure variation along the fiber radius, $\rho_{\mathrm{f}}$. Which range of scattering angles is covered by the pattern is, in principle, irrelevant. Though we are concerned with small-angle X-ray scattering (SAXS) patterns, the effects considered here can be applied to the range of the ultrasmall-angle X-ray scattering (USAXS) without loss of generality and to the range of the middle-angle X-ray scattering (MAXS) in good approximation. In wide-angle X-ray scattering (WAXS) the geometric relations are more complex, because there the curvature of the Ewald sphere has to be considered.

In previous work ${ }^{21}$ we have established a tomographic method for the desmearing of sequences of scattering patterns recorded in microbeam scans by one-dimensional tomography. ${ }^{22,23}$ From the point of view of an engineer it may be argued that a method that does not require rotation of the sample was not tomography. The scientist identifies the common abstract principle of general tomography and one-dimensional tomography. Both methods retrieve volume ("slice") information from projected data by application of the Fourier-slice theorem.

In our reconstruction ${ }^{21}$ we have assumed that the scattering from every local volume element ("voxel") $V\left(\rho_{\mathrm{f}}\right)$ exhibits fiber symmetry. The size of the voxel is defined by the size of the microbeam, and $\rho_{\mathrm{f}}$ is the distance of the voxel from the fiber axis. The assumption does not hold, in general. This fact is established in the analysis of new scattering patterns. As a consequence, the visibility of structural features in the scattering patterns is confined or shifted to a zone of the fiber from which they do not originate. In a methodical discussion, we demonstrate how to benefit from the nonideal character in order to gain extra information on the nanostructure of cylindrical samples.

It is an aim of this feasibility study to extract quantitative information on structure gradients both from the raw patterns (in projection space) and from reconstructed patterns (in image space), to compare them, and to assess the significance of simple 


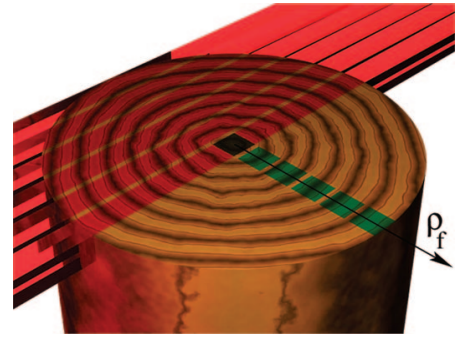

Figure 1. A fiber is scanned by an X-ray micobeam (left). The beam integrates structure from various annular zones in the fiber. Perfect tomographic reconstruction returns the scattering that emanates from single voxels (right, squares), $V\left(\rho_{\mathrm{f}}\right)$, on the fiber radius, $\rho_{\mathrm{f}}$.

methods of parameter extraction. Among these methodical aims, we are trying to answer some questions related to application. Thus, additional studies of the neat components are carried out in order to ease the assignment of features in the patterns of the MFC (e.g., is there scattering of semicrystalline poly(ethylene terephthalate) (PET)?). We are aiming to identify the scattering effect of the microfibrillar PET component. After tomographic reconstruction, we expect to be able to assign superimposed features of the scattering patterns (point diagrams, rings, streaks) to distinct zones in the fiber cross section and to study their variation along the fiber radius.

A very recent study ${ }^{24}$ has been brought to our knowledge by one of the reviewers. It is aiming at an elucidation of the radial structure gradient of fibers by means of a sophisticated engineering approach (a so-called "on-axis" experiment). Filament sections of $15 \mu \mathrm{m}$ length are prepared by laser dissection and scanned by a microbeam that is running parallel to the axis of the sample. Considering the geometry of this setup, the resulting scattering patterns are made from the scattering intensity in the equatorial plane of the irradiated voxel (400 $\mathrm{nm} \times 400 \mathrm{~nm} \times 15 \mu \mathrm{m})$ that contains information on the projection of Bonart's "transversal structure" 25,26 on the crosssectional plane of the filament. Thus, in an on-axis experiment its spatial variation is only subjected to smearing along the sample length. Because smearing across the filament diameter is avoided, the respective tomographic reconstruction is unnecessary. Nevertheless, a quantitative analysis ${ }^{27}$ appears to be problematic, because in the chosen geometry scattering data have become inaccessible that would be required to convert the corresponding real-space information from a projection into a section. As long as the nanostructure of the filament is conforming to a microfibrillar model ${ }^{28}$ and the irradiated volume is too big to resolve the inner structure of a microfibril, one expects that each "on-axis" SAXS pattern shows pseudoisotropy. Indeed, in the mentioned study this is found with most of the patterns, except for patterns from a thin shell zone of the filament. There an intensity streak is found that is extending in radial direction of the filament. Anticipating one of our results, we have found a very similar feature in some of our strands which are thicker by several orders of magnitude and have associated it to "shingle voids".

\section{Theoretical: Visibility of Structure in X-ray-Scattering Microbeam-Scanning Experiments of Fibers}

2.1. Overview. Figure 1 sketches $X$-ray microbeam-scanning of a polymer fiber and the target of tomographic reconstruction, namely the reduction of the integrated structure information swept out by the beam to the inherent information of single voxels. We assume that the structure does not change in annular zones around the fiber axis. In this case, rotation of the fiber about its axis does not change the measured data. A single scan is collecting all the accessible information for a structure analysis with spatial resolution.

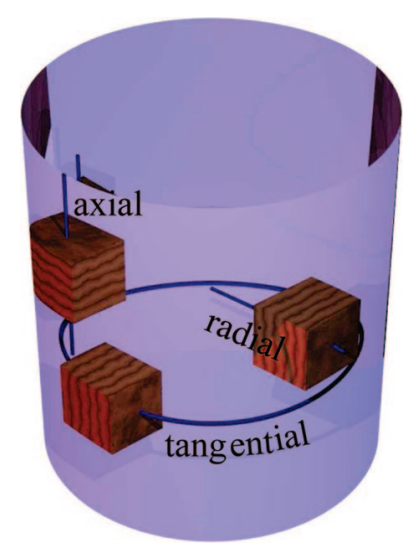

Figure 2. Definition of elementary local grains inside a fiber. Only a voxel with axial grain exhibits local fiber symmetry with respect to the macroscopic fiber axis.

For fibers, even the tomographic reconstruction technique is considerably simplified, resulting in one-dimensional tomography. ${ }^{22}$ In principle, this technique is nothing but onion peeling. ${ }^{21,29}$ The border pattern from the microbeam scan is only containing information on the outer shell-zone of the fiber and may be peeled off. This principle is readily iterated. The related integral transform has been deduced in 1826 by Niels Abel. ${ }^{30}$

Perfect reconstruction is possible, if a scalar quantity (e.g., the absorption) is recorded at each scan position. Utilizing the inverse Abel transform ${ }^{30-32}$ fast, low-noise algorithms ${ }^{22,23}$ are made available. They reconstruct the absorption $A\left(\rho_{\mathrm{f}}\right)$ existing in image space $\rho_{\mathrm{f}}$ (along the fiber radius) from the measured projected absorption $\{A\}(x)$ in projection space $x$.

In our case, data of higher dimensionality are collected at every scan position $x$ outside the fiber. Even if a twodimensional scattering pattern, $\{I\}(s, x)$, is collected, some of $\mathrm{us}^{21}$ have proposed to utilize the inverse Abel transformation

$$
I\left(\mathbf{s}, \rho_{\mathrm{f}}\right)=-\frac{1}{\pi} \int_{\rho_{\mathrm{f}}}^{\infty} \frac{\mathrm{d}\{I\}(\mathbf{s}, r)}{\mathrm{d} r} \frac{\mathrm{d} r}{\sqrt{r^{2}-\rho_{\mathrm{f}}^{2}}}
$$

in order to reconstruct a desmeared scattering pattern along the fiber radius $\rho_{\mathrm{f}}$ tomographically (X-ray scattering fiber computertomography (XSF-CT)). However, in this case perfect reconstruction is only possible if the local structure of each voxel $V\left(\rho_{\mathrm{f}}\right)$ generates a scattering pattern $I(\mathbf{s})=I\left(s_{12}, s_{3}\right)$ with fiber symmetry. Here $\mathbf{s}$ is the scattering vector. Its modulus is defined $s=(2 / \lambda) \sin \theta \cdot \lambda$ is the wavelength of the $\mathrm{X}$-rays and $2 \theta$ is the scattering angle.

Anticipating the following discussion, deviations from local fiber symmetry (LFS) cause striking effects in scattering patterns from scanning-microbeam experiments. This means that an expectation expressed in an earlier paper ${ }^{33}$ is not justified, namely "that fiber symmetry should have been imprinted on the contents of every voxel by averaging, because in tomography the sample is rotated about an axis-in analogy to the classical rotationcrystal method".

Deviations from LFS cause restricted or shifted visibility of scattering features along the fiber radius, thus causing aberration in the tomographic reconstruction. In more detail, structural entities with tangential grain (cf. Figure 2) are only visible in the central scattering pattern (as long as the step width is not less than the integral width of the microbeam), regardless of the zone in which they are placed. XSF-CT is accumulating their structure additionally in the central voxel of the image (infra-reconstruction). Thus, there are special rules for the interpretation of the central scattering pattern.

If structural entities in a fiber zone are carrying radial grain (i.e., annular character, cf. Figure 2), their visibility is consider- 


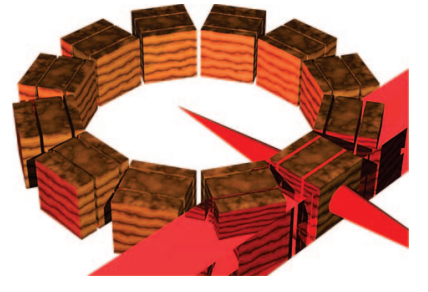

Figure 3. A fiber zone modeled by a ring of cubic voxels. All voxels exhibit axial grain plus some solitary shingle voids in tangential orientation. The axial grain is always visible to the scanning microbeam. The shingles, on the other hand, are only seen when they are parallel to the beam. Cones sketch the streak-scattering of the shingles.

ably restricted. As a result, the XSF-CT is wrongly peeling-off their scattering effect from all the inward zones. We call this effect ultrareconstruction. Aberrations caused from ultrareconstruction can be eliminated by removing the generating scattering feature from the pattern in which it becomes visible.

2.2. General Visibility of Various Scattering Features. The orientation distribution of structural entities is controlling their visibility in scanning-microbeam experiments of fibers. Perfectly reconstructible are isotropic features and features with fiber symmetry parallel to the fiber axis (axial grain). They exhibit the same scattering effect in every pattern along the fiber scan.

Visible are structural entities whose scattering pattern is in the detector plane. All the layer-shaped patterns of rodlike entities (e.g., needle-shaped voids, microfibrils, or ensembles of such domains) are generally visible (because two planes in space generally share a common line). We assume that visibility variation of rodlike entities may be disregarded in many technical polymer materials.

Frequently invisible are the streak-shaped scattering patterns generated by extended layers. The reason is that the streak scattering is, in general, not in the detector plane. Nevertheless, there is at least one pattern from a fiber-scanning microbeam experiment in which the streak flares up. Strong streak scattering may even be caused from sparse and uncorrelated layers in the voxel, if the layers are voids. Let us call such entities shingle voids.

As layers are arranged on top of each other, they cross each voxel like wood grain. In analogy to the orientation of a solitary layer, now the orientation of the grain is controlling visibility and reconstructibility in XSF-CT.

2.3. Axial Grain and Perfect Reconstruction. Layer stacks or tapered microfibrils in fibers are frequently oriented in such a way that the density variation is strongest in the direction of the macroscopic fiber axis. This is the case which reconstructs perfectly in XSF-CT. Figure 3 sketches the ideal case in conjunction with the most frequently observed aberration. A ring-shaped zone of the fiber is shown. As the microbeam is translated across the fiber, it provokes the same scattering effect (a two-point pattern) in every voxel from the considered zone, in principle. Thus, tomographic reconstruction of the lamellae structure is perfect.

On the other hand, the shingle voids from this zone become only visible when the microbeam is grazing the zone (as sketched in Figure 3). Only in this case the streak scattering of the shingle voids is in the detector plane. It is worth mentioning that a shingle-void streak must be observable as well in an onaxis experiment, ${ }^{24}$ whereas the scattering effect of an extended needle would simply add to the pseudoisotropic component of the corresponding pattern. Thus, a reported ${ }^{24}$ observation of such streaks in the shell zone of filaments is in agreement with our explanation.

2.4. Radial Grain and Ultrareconstruction. The argument just brought forward for a solitary shingle also holds for a stack
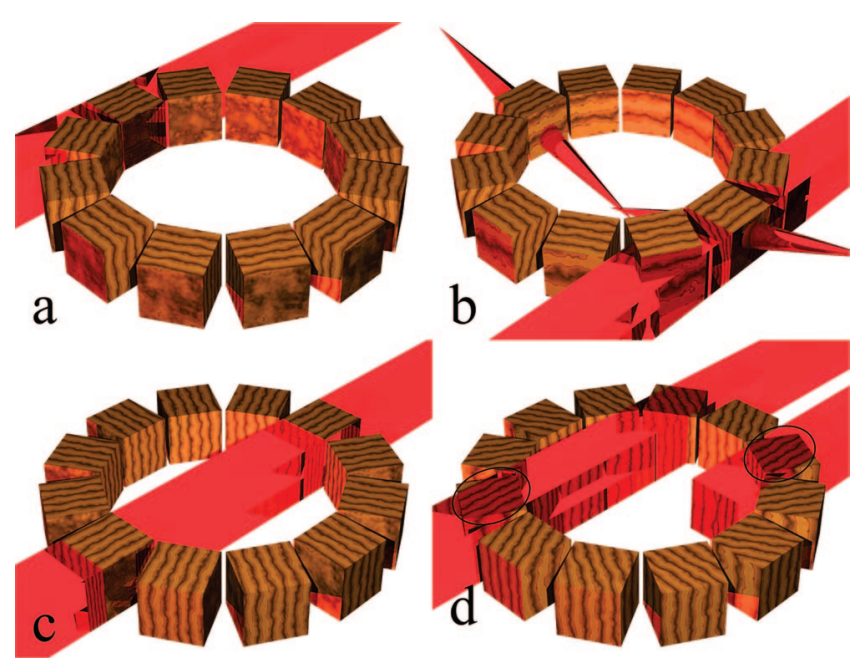

Figure 4. Fiber zone sketched by a ring of voxels. Elementary deviations from local fiber symmetry are displayed. Microbeams indicate the scan positions, at which the otherwise hidden structures become visible. (a) Radial grain. (b) Tilted radial grain. Cones indicate the tilt of the apparent equator "plane". (c) Tangential grain. (d) Turned grain.

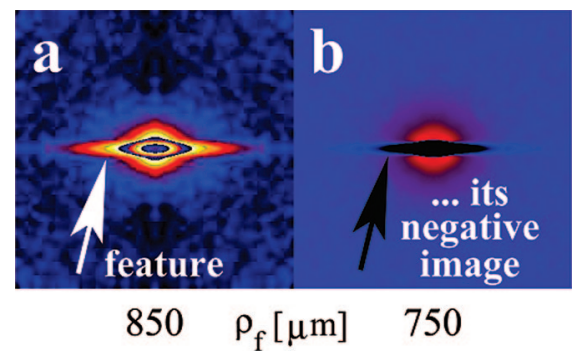

Figure 5. Demonstration of the ultrareconstruction artifact in tomographic reconstructions of nonscalar quantities: (a) arrow pointing at a real streak feature in an outer zone of the irradiated sample; (b) reconstructed negative intensity in an inner zone with the shape of a feature from an outer zone.

of layers with its normal pointing in radial direction. Again, it is only detected by the grazing microbeam (Figure 4a). Because XSF-CT assumes visibility of the corresponding scattering pattern in the complete ring, tomographic imaging results in the artifact of ultrareconstruction. Thus, already invisible features are compensated a second time. This overreconstruction is affecting inward zones of the tomogram, where the scattering intensity frequently even becomes negative-similar to the known effect of overdesmearing that is probed in blind deconvolution. ${ }^{34-36}$ The effect is demonstrated in Figure 5. If radial grain is additionally tilted with respect to the fiber direction (Figure 4b), it is readily detected while watching the microbeam experiment: From one image to the next a sudden tilt of the equator "plane" is observed, or the new pattern is showing a superposition of two patterns with differing equatorial "plane".

Whenever the intensity depression of ultrareconstruction is detected in a zone $\rho_{f}^{\prime}$, the corresponding scattering feature is located in the neighborhood at $\rho_{\mathrm{f}}>\rho_{\mathrm{f}}^{\prime}$. The shape of the depression indicates the causing scattering entity: a streak indicates shingle voids, and a two-point diagram indicates stacks of lamellae. In our first paper on SAXS tomography, ${ }^{33}$ we had been puzzled by such depressions, which now are explained by stacks of tilted lamellae.

After ultrareconstruction has been detected in the reconstructed scattering data, there are several possibilities to remove the corresponding perturbation in a refinement step. If the 
Table 1. Studied Samples of Varying Cold-Draw Ratio $\lambda_{d}$ and Composition $^{a}$

\begin{tabular}{cccc}
\hline$\lambda_{\mathrm{d}}$ & 1 & 3 & 7 \\
\hline PET & $\times$ & & $\times$ \\
PEBA & $\times$ & $\times$ & $\times$ \\
MFC & & $\times$ \\
${ }^{a}$ MFC is made by co-extrusion of $70 \mathrm{wt} \%$ PEBA and $30 \mathrm{wt} \%$ PET.
\end{tabular}

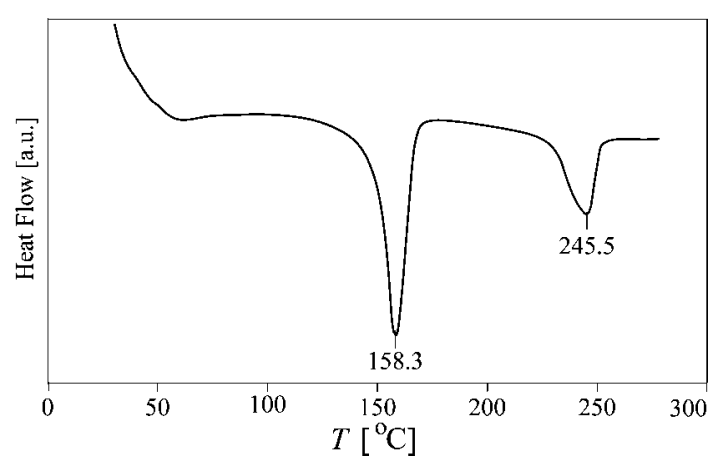

Figure 6. Differential-scanning calorimetry melting curve of sample MFC $\left(\lambda_{\mathrm{d}} \approx 7\right)$ (i.e., the composite PEBA/PET 70/30 (by weight)) (instrument: DSC Q1000, TA Instruments Inc.).

scattering from the outmost shell zone is only caused by radial grain, the corresponding scattering pattern is simply removed from the set of data. Thus, disturbance of the inner zones is avoided. If the aberration is resulting in only a diffuse depression, it may be corrected by spatial frequency filtering. ${ }^{33}$ Slight indentations restricted to a narrow central area in the patterns may be removed by cut and extrapolate. If the scattering effect of the disturbance is not diffuse, it can be interpreted and separated. The extracted negative pattern can be adjusted to compensate the effect in the causing zone.

2.5. Tangential Grain and Infrareconstruction. If a zone of a fiber contains structural entities with tangential density variation (Figure 4c), it is only visible when the microbeam hits the axis of the fiber. Thus, only the measured central scattering pattern has accumulated all the corresponding features. Consequently, even the reconstructed XSF-CT pattern rarely presents the structure from only the fiber center. Negligibility of infrareconstruction can be detected by comparing the reconstructed central pattern with the patterns of its neighbors. If the central (on-axis) pattern appears to be the smooth continuation of the sequence of off-axis patterns, infrareconstruction due to voxels with tangential grain appears to be negligible. The intermediate state between tangential and radial grain is the turned grain presented in Figure 4d. Such structural entities are not mapped on the center but are shifted toward the center and appear in a different zone. In all the zones lying even more inward, turned grain will cause ultrareconstruction as XSF-CT is applied.

\section{Experimental Section}

3.1. Materials. The studied samples are the starting components and the precursor materials from laboratory-scale production of microfibrillar-reinforced composites made from poly(ethylene terephthalate) (PET) and a poly(ether)-block-amide (PEBA). The PET grade is a commercial product ("Laser C B95A" by DuPont Inc., USA) with a melting temperature $T_{\mathrm{m}}=236{ }^{\circ} \mathrm{C}$. The PEBA is a commercial product ("PEBAX 7233" by Arkema Inc., France), $T_{\mathrm{m}}=158{ }^{\circ} \mathrm{C}$. The reported melting points have been determined by differential scanning calorimetry of the sample MFC (cf. Table 1 ) as the minimum endotherm values (cf. Figure 6).

All samples are extruded cylindrical strands of diameters between 1 and $2 \mathrm{~mm}$ that were prepared as follows. The blend was predried (at $100{ }^{\circ} \mathrm{C}$ for $48 \mathrm{~h}$ ). Extrusion was carried out on a single-screw extruder BX-18 (Axon AB, Sweden) with the following temperature profile: zone 1 (next to the hopper) $250{ }^{\circ} \mathrm{C}$, zone 2 and $3260{ }^{\circ} \mathrm{C}$, zone $4270{ }^{\circ} \mathrm{C}$, die $1250{ }^{\circ} \mathrm{C}$, die $2238{ }^{\circ} \mathrm{C}$. Diameter of the die was $1 \mathrm{~mm}$. Screw rotation speed was $36 \mathrm{rpm}$. After extrusion, some of the samples were cold-drawn (at $60{ }^{\circ} \mathrm{C}$ ) to different draw ratios $\lambda_{\mathrm{d}}=l / l_{0}$ with $l_{0}$ the original length of the strand after extrusion and $l$ the length of the sample after cold drawing (cf. Table 1). None of the samples was subjected to the final thermal treatment step, which is typical for the production of an MFC with isotropic matrix phase. In other words, the studied samples represent components or precursors (drawn blend) for manufacturing of MFC.

3.2. Setup. Scanning-microbeam SAXS experiments are carried out at HASYLAB, Hamburg, beamline BW4. The incident primary beam (wavelength $\lambda=0.13 \mathrm{~nm}$ ) is focused by means of a stack of Be-lenses ${ }^{37,38}$ yielding a beam cross section at the sample of 40 $\mu \mathrm{m}$ integral width and $39 \mu \mathrm{m}$ height as measured by a knife edge. The strands are linearly scanned through the beam with a step size of $50 \mu \mathrm{m}$. The distance between sample and detector is $1910 \mathrm{~mm}$. Each scattering pattern is exposed for $40 \mathrm{~s}$ using a 2D marccd 165 detector (mar research, Norderstedt, Germany). A low-noise machine background pattern is exposed for $3 \mathrm{~min}$. The absorption of the primary beam is measured by monitoring the beam intensity before and after the sample.

A simple check for fiber symmetry was carried out by performing a second scan after rotating the fiber by $90^{\circ}$ about its axis and comparing the results. In all cases the pairs of scans are identical. Surface scattering or beam refraction effects at the scan extremities are not observed.

Pre-evaluation. The measured machine background pattern is subtracted from each microbeam-scan pattern after weighting by the measured absorption factor. No normalization to constant sample thickness is performed. Further pre-evaluation steps follow the standard method. ${ }^{39}$ The images are centered and aligned, and some blind spots can be filled from symmetry consideration. The remnant hole in the center $\left(|s|<0.018 \mathrm{~nm}^{-1}\right)$ is filled by $2 \mathrm{D}$ igloo-tent extrapolation. ${ }^{36}$ Data from a quadratic area $\left(-0.2 \mathrm{~nm}^{-1}<s_{12}, s_{3}<\right.$ $0.2 \mathrm{~nm}^{-1}$ ) are kept for further evaluation because outside of this area there is no relevant scattering.

For tomographic image reconstruction the center of the fiber is determined and the images are interpolated accordingly. Thus, the first image in the set of patterns becomes the pattern related to a central irradiation of the fiber. The XSF-CT reconstruction is accomplished by a matrix-vector multiplication. ${ }^{21}$ Thus, the reconstruction method is defined by the reconstruction matrix. We have applied two different reconstruction methods, which differ by the amount of noise that they return as a function of the noise in the input data. The two-point Abel inversion after $\operatorname{Dasch}^{22}$ appears more appropriate for the reconstruction of weak scattering patterns from microbeam scans. Stronger scattering patterns are more smoothly reconstructed by the BASEX ${ }^{23}$ method.

\section{Results and Discussion}

4.1. PET $\lambda_{\mathbf{d}}=1$. The PET precursor material (as extruded, i.e. $\lambda_{\mathrm{d}}=1$ ) is a strand of $1.8 \mathrm{~mm}$ diameter. The SAXS pattern recorded with a macrobeam of $3 \mathrm{~mm}$ width exhibits diffuse, isotropic scattering and a weak but sharp equatorial streak. It is pictured at the bottom of Figure 7. Thus, the material appears amorphous with some voids. The voids appear to be highly oriented in the fiber direction. A lower limit of the void height may be estimated from the height of the equatorial streak. From the length of the streak one would probably estimate a void diameter. Thus, one would implicitly assume that the voids exhibit the shape of needles.

In the scanning microbeam (Figure 7), the PET exhibits a striking sequence of scattering patterns: only in a thin outer shell the sharp equatorial streak is observed, although it should be visible in all scattering patterns under the premise of LFS. The experiment demonstrates that here the condition of LFS is not fulfilled. Subjected to XSF-CT reconstruction, a negative image 


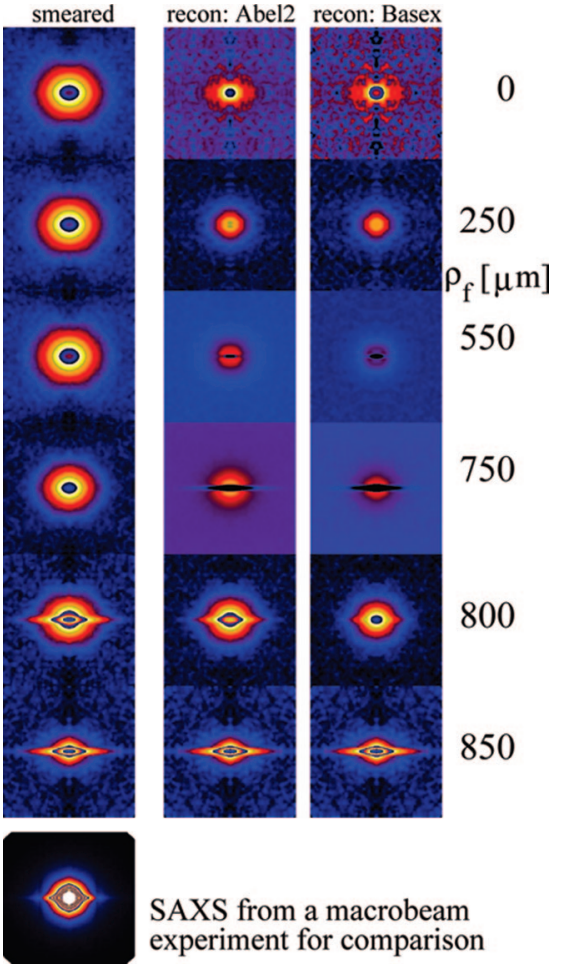

Figure 7. SAXS patterns of a PET strand (as extruded, $\left(\lambda_{\mathrm{d}}=1\right)$ ) in a scanning microbeam experiment. Logarithmic intensity scale. The fiber direction $s_{3}$ is vertical. Transverse direction: $s_{12}$. All patterns display the range $-0.2 \mathrm{~nm}^{-1} \leq s_{12}, s_{3} \leq 0.2 \mathrm{~nm}^{-1}$. Within each column the scale is kept constant. Left column: measured patterns. The other columns show the reconstructed patterns after XSF-CT as a function of the distance $\rho_{\mathrm{f}}$ from the axis of the strand for two different reconstruction methods. The bottom pattern shows the result of a macrobeam SAXS experiment carried out in 2004 with a worn-out detector. The dark vertical bars are detector artifacts.

of the streak shows up in several inner zones. For the undrawn PET strand (Figure 7), this effect is clearly detected between $\rho_{\mathrm{f}}$ $=550 \mu \mathrm{m}$ and $\rho_{\mathrm{f}}=750 \mu \mathrm{m}$. For shorter $\rho_{\mathrm{f}}$ the fraction of the shell volume in the volume swept out by the microbeam is so small that the aberration effect carries little weight.

If the observed equatorial "streak" were caused from needleshaped voids, it would be visible throughout the scan because the scattering of oriented needles exhibits LFS. Consequently, the scattering entities cannot be needles. The observed phenomenon is smoothly explained by assuming that the voids look like shingles with their normals oriented in radial fiber direction (cf. Figure 3). In the course of data evaluation, we have used the "equatorial streak" at the left side of the fiber scan to determine the principal axis of the scattering pattern. At the right side of the fiber scan we, again, observe a streak, but its direction is tilted by $10^{\circ}$ with respect to the streak observed at the opposite side. Figure $4 \mathrm{~b}$ demonstrates, how this additional effect is explained by tilt of the shingle planes.

Comparison of the reconstructed pattern of the central voxel $\left(\rho_{\mathrm{f}}=0\right)$ with its neighbors $\left(\rho_{\mathrm{f}}>0\right)$ shows here and in most of the other microbeam scans completely different patterns. The severe distortion of the central pattern is readily explained by the assumption that some zones of the fiber contain structural entities with tangential grain (cf. Figure 4c) that are mapped on the central voxel because of infrareconstruction.

4.2. PET $\lambda_{\mathrm{d}}=$ 7. After the PET strand is cold-drawn to $\lambda_{\mathrm{d}}$ $\approx 7$, it shows a homogeneous structure in the microbeam scan. There is no strong equatorial streak. All the scan positions exhibit the same diffuse scattering (Figure 8). However, as compared to the undrawn material now the diffuse scattering

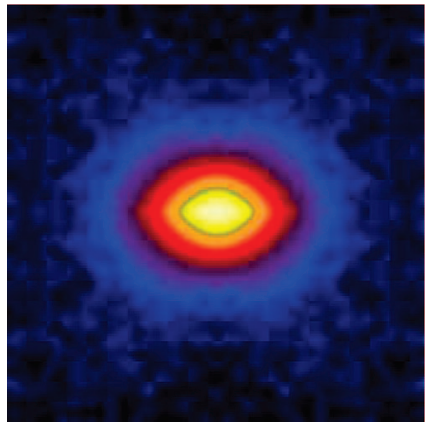

Figure 8. SAXS pattern of a neat PET strand cold-drawn to $\lambda_{\mathrm{d}} \approx 7$ recorded in a microbeam experiment at all positions. Logarithmic intensity scale. The fiber direction $s_{3}$ is vertical. The pattern shows the range $-0.2 \mathrm{~nm}^{-1} \leq s_{12}, s_{3} \leq 0.2 \mathrm{~nm}^{-1}$.

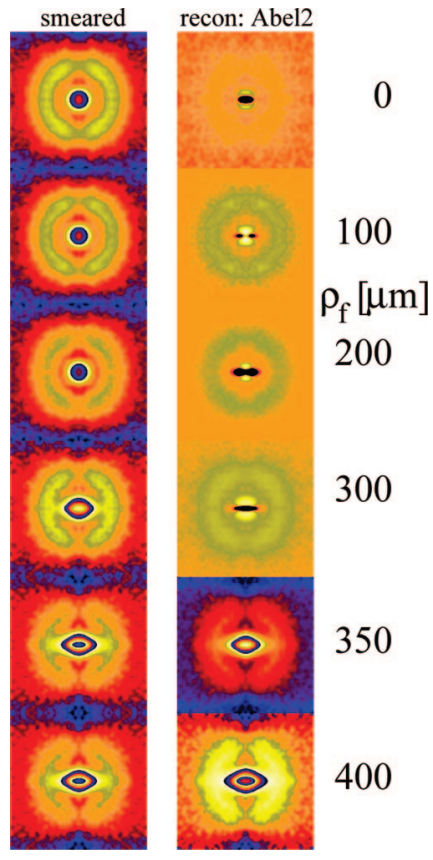

Figure 9. SAXS patterns of a PEBA strand "as extruded" $\left(\lambda_{d}=1\right)$ (diameter $=0.9 \mathrm{~mm}$ ) in a scanning-microbeam experiment. Logarithmic intensity scaling. The fiber direction $s_{3}$ is vertical. The patterns display the range $-0.2 \mathrm{~nm}^{-1} \leq s_{12}, s_{3} \leq 0.2 \mathrm{~nm}^{-1}$. The scaling is constant within a column. Left column: measured scanning-microbeam patterns. Right column: XSF-CT reconstructed patterns as a function of the distance $\rho_{\mathrm{f}}$ from the axis of the strand.

has become slightly anisotropic: The contour lines are no more circles, but ellipses with an aspect ratio of 1.5 . We do not observe a long period reflection of semicrystalline PET.

4.3. PEBA $\lambda_{\mathbf{d}}=1$. All strands made from neat PEBA are weak scatterers. The patterns exposed for $40 \mathrm{~s}$ have been smoothed considerably. Thus, they are insufficient for quantitative analysis. Figure 9 shows that even the as-extruded material is oriented. All patterns from the microbeam scan show a short equatorial streak and strong, arc-shaped reflections at the equator with a long period of $10 \mathrm{~nm}$. Thus, the hard domains of the PEBA appear arranged preferentially transverse to the fiber direction. The tomographic analysis returns a more detailed view. Again, the central voxel suffers from strong infrareconstruction. Up to $\rho_{\mathrm{f}}=300 \mu \mathrm{m}$ there is no orientation. The isotropic long period is $10 \mathrm{~nm}$. Here negative-intensity equatorial streaks indicate overreconstruction. Thus, at least a fraction of the equatorial streak observed further out must be attributed to shingle-shaped voids in radial orientation. Utilizing a finer microbeam, it would be possible to distinguish if the isotropic and the anisotropic zones are separated by a shingle-void zone. 

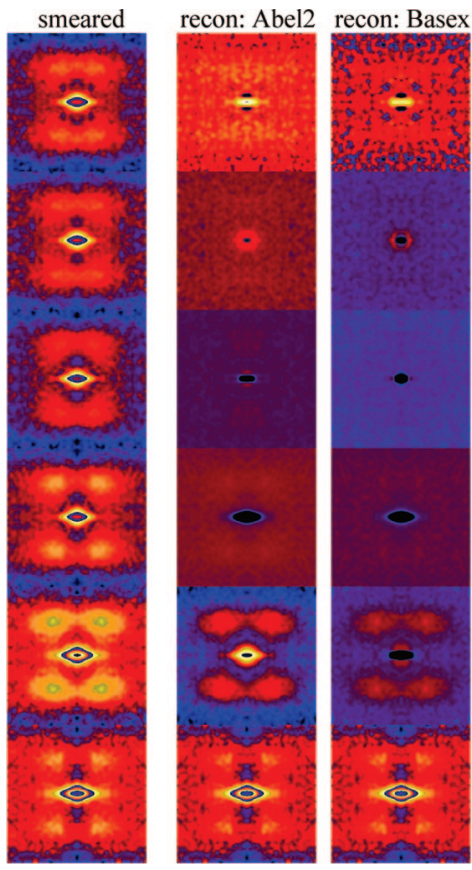

Figure 10. SAXS patterns of a cold-drawn $\left(\lambda_{\mathrm{d}} \approx 7\right)$ PEBA strand (diameter $=1 \mathrm{~mm}$ ) in a scanning-microbeam experiment. Logarithmic intensity scaling. The fiber direction $s_{3}$ is vertical. The patterns display the range $-0.2 \mathrm{~nm}^{-1} \leq s_{12}, s_{3} \leq 0.2 \mathrm{~nm}^{-1}$. The scaling is constant within a column. Left column: measured scanning-microbeam patterns. Middle and right columns: XSF-CT reconstructed patterns (two different reconstruction methods) as a function of the distance $\rho_{\mathrm{f}}$ from the axis of the strand.

4.4. PEBA $\lambda_{d}=7$. The weak scattering data of the 7 -fold drawn strand from neat PEBA (Figure 10) suffice for a qualitative interpretation only. In order to record good patterns using the available setup, the exposure would have to be increased by a factor of 10 . Comparison of the two reconstruction methods shows that here the two-point Abel inversion returns less noise than the BASEX method. Due to the postprocessing (smoothing of more noisy data) the peaks of the BASEX-reconstructed patterns appear weaker than those of the Abel-reconstructed patterns. We observe inversion of the reported $^{21}$ advantage of the BASEX method, if the smeared measured data carry considerable noise.

In all recorded patterns from the microbeam scan we observe a 4-point pattern with its peak maxima always found at $\left(s_{12 L}, s_{3 L}\right)$ $=\left( \pm 0.052 \mathrm{~nm}^{-1}, \pm 0.093 \mathrm{~nm}^{-1}\right)$. The tomographic reconstruction exhibits that this feature is generated only in a shell zone of the strand, which is approximately $100 \mu \mathrm{m}$ wide. The observed pattern can be explained by a macro lattice ${ }^{40}$ of narrow block stacks, in which the longitudinal distance between the hard-domain blocks is $1 / s_{3 L}=11 \mathrm{~nm}$. These block stacks can be addressed as microfibrils, which are not homogeneous needles but linear arrangements of alternating hard domains and soft domains, respectively. The transverse distance between these PEBA microfibrils is $1 / s_{12 L}=19 \mathrm{~nm}$.

A different explanation by a system of tilted lamellae stacks cannot be excluded completely, because the data are so noisy. If we choose the latter model for explanation, the stacks are tilted by $\pm 30^{\circ}$ with respect to the meridian and exhibit a long period of $9 \mathrm{~nm}$. Nevertheless, in the core of the fiber we do not observe ultrareconstruction in the shape of a 4-point diagram, which would prove the presence of tilted lamellae. An example of such ultrareconstruction was first observed by us with a polyethylene strand. ${ }^{33}$ Because stacks of tilted lamellae show grain, whereas macrolattices from block stacks do not, tilted lamellae may violate LFS and, thus, may cause overreconstruction.

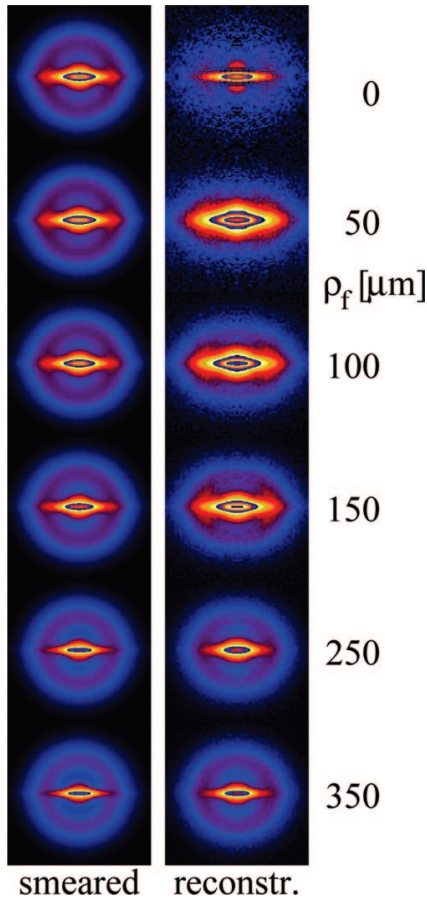

Figure 11. Cold-drawn $\left(\lambda_{\mathrm{d}} \approx 3\right) \mathrm{MFC}$ in a scanning microbeam experiment. Measured scattering intensity $\{I\}\left(s_{12}, s_{3}, \rho_{\mathrm{f}}\right)$ (left column) and reconstructed scattering $I\left(s_{12}, s_{3}, \rho_{\mathrm{f}}\right)$ (right) for short distances $\rho_{\mathrm{f}}$ from the fiber axis. The patterns display the range $-0.1 \mathrm{~nm}^{-1} \leq s_{12}, s_{3} \leq 0.1$ $\mathrm{nm}^{-1}$ in uniform logarithmic scale.

Admittedly, there was a small chance that the reported nonexistence of scattering were a reconstruction artifact, if a very special nanostructure would exist. One would have to assume superposition of two scattering entities that match with regard to both their repeat units and their volume fractions as a function of $\rho_{\mathrm{f}}$ and annihilate under XSF-CT reconstruction. A probable example was stacks of tilted lamellae that exhibit LFS plus a fraction that does not. For ultrareconstruction to occur, the normals of the non-LFS lamellae stacks in every voxel must preferentially be tilted toward and away from the fiber axis in each voxel from the voxel ring.

The central voxel shows distinct infrareconstruction caused by the accumulation of all structural entities with tangential grain. There is no void scattering. Ultrareconstruction is weak and restricted to a narrow zone in the center of the reconstructed scattering patterns. We cannot exclude that this ultrareconstruction and the visible differences between the two reconstructions are caused from the considerable noise in the original data.

4.5. MFC $\lambda_{\mathbf{d}}=3$. MFC is our abbreviation for the colddrawn coextrudate of $70 \mathrm{wt} \%$ PEBA and $30 \mathrm{wt} \%$ PET. The material presented in this section was cold-drawn to $\lambda_{\mathrm{d}} \approx 3$. Its scattering intensity is by a factor of 20 higher than that of neat PEBA. In the scanning-microbeam experiment, the strand shows an isotropic long period and an equatorial streak at almost every beam position (Figure 11, left column). Only the tomographically reconstructed patterns (right column) exhibit that the longperiod ring reflection is not existent in the core of the fiber. As the reflection becomes visible, it first shows up at the equator. With increasing distance from the fiber axis, reflection arcs are growing toward the meridian. Above $\rho_{\mathrm{f}}=300 \mu \mathrm{m}$ the arcs join into a closed circle. We have seen this behavior before with the neat PEBA (cf. Figure 9). Thus, this phenomenon is not indicating some interaction between the PEBA and the PET microfibrils. On the other hand, with the MFC the isotropization is proceeding outward from the center, whereas this has been just opposite with the neat PEBA strand (Figure 9). The reconstructed central voxel $\left(\rho_{\mathrm{f}}=0\right)$ clearly exhibits infrarecon- 


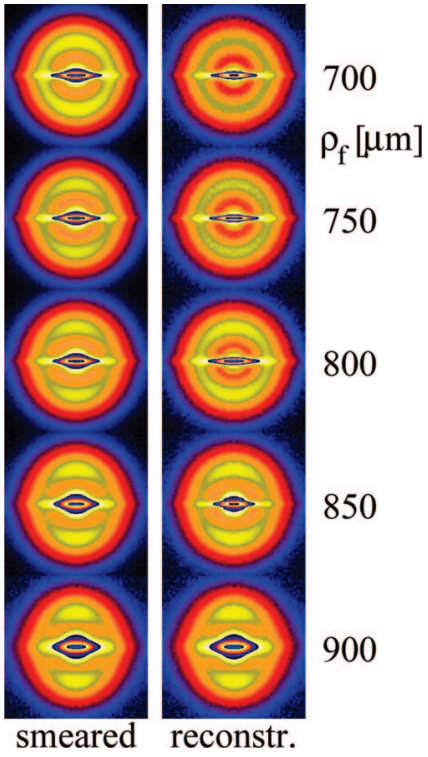

Figure 12. Cold-drawn $\left(\lambda_{\mathrm{d}} \approx 3\right)$ MFC in a scanning microbeam experiment. Measured scattering intensity $\{I\}\left(s_{12}, s_{3}, \rho_{\mathrm{f}}\right)$ (left column) and reconstructed scattering $I\left(s_{12}, s_{3}, \rho_{\mathrm{f}}\right)$ (right) for long distances $\rho_{\mathrm{f}}$ from the fiber axis. The patterns display the range $-0.1 \mathrm{~nm}^{-1} \leq s_{12}, s_{3} \leq 0.1$ $\mathrm{nm}^{-1}$ in uniform logarithmic scale.

struction. Ultrareconstruction is not observed in this strand. Examination of the equatorial streak exhibits only in the reconstruction that it grows broader toward the center of the fiber. Because we do not observe ultrareconstruction we allocate the streak to needle-shaped domains, which we have not seen with any of the strands made from neat polymers. If these needles are thin PET microfibrils, the tomography shows that in the center of the fiber these microfibrils are shorter than in the middle of the radius. The right column shows the reconstruction result of the BASEX algorithm. With this strongly scattering material, the pseudocolor images of Abel and BASEX algorithm cannot be distinguished from each other. However, now the BASEX method returns the result with less noise.

Between $\rho_{\mathrm{f}}=350 \mu \mathrm{m}$ and $\rho_{\mathrm{f}}=700 \mu \mathrm{m}$ we do not observe qualitative change in the patterns. Thus, we turn to the outer zones of the MFC strand. Figure 12 displays the corresponding variation of the scattering. At the fiber surface $\left(\rho_{\mathrm{f}}=900 \mu \mathrm{m}\right)$ the long-period reflection of the PEBA is highly oriented in fiber direction. Its considerable extension in lateral direction indicates that the scattering domains are narrow in lateral direction. Thus, we do not observe stacks of lamellae, but stacks made of narrow blocks. We have called these stacks PEBA microfibrils with the neat material. As we track the orientation of the block stacks inward, the reconstructed patterns show that the PEBA phase has already become isotropic after $150 \mu \mathrm{m}$. A considerable change of the scattering power is not observed. Thus, the population density with block stacks does not change considerably. Probably this anisotropic shell zone can easily be turned isotropic by annealing, in order to generate the isotropic matrix phase of an ideal MFC.

Close to the fiber surface the equatorial streak is rather broad. The tomographic reconstruction reveals that it has turned slim already $50 \mu \mathrm{m}$ below the surface. Ultrareconstruction is not observed. Thus, the streak is associated with the scattering of highly oriented and long needle-shaped microfibrils. Only in the very thin outer shell zone the needles are shorter. Augmenting this observation with a similar finding from the central region of the strand (Figure 11), we subsume that shorter microfibrils are found in the center and at the surface of the strand.

For a quantitative evaluation the isotropic long-period feature must be separated from the equatorial streak. For this purpose

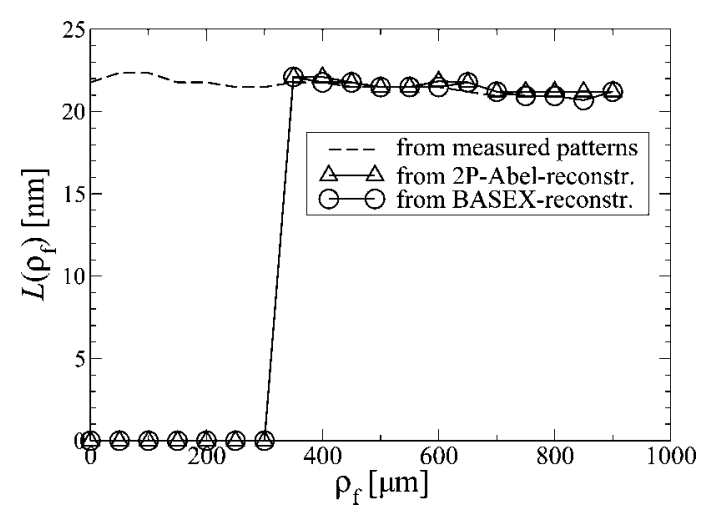

Figure 13. $\operatorname{MFC}\left(\lambda_{\mathrm{d}} \approx 3\right)$ in the scanning microbeam experiment. Spatial variation of the long period $L\left(\rho_{\mathrm{f}}\right)$, as determined from the position of the ring maximum. $\rho_{\mathrm{f}}$ is the distance from the fiber axis. The dashed curve shows data determined from the measured data. The solid lines present the results obtained after tomographic reconstruction.

the superimposed scattering has been suppressed in an angular region of $\pm 45^{\circ}$ around the equator. By azimuthal averaging of the rest, the scattering of the isotropic long period feature, $I_{i}(s)$, is retrieved. The long period is then determined by the position of the maximum $s_{L}$ using the relation $L=1 / s_{L}$. Figure 13 shows the result. The value is slightly decreasing with increasing $\rho_{\mathrm{f}}$ and does not change upon tomographic reconstruction. Of course, in projection space (measured patterns) a ring reflection can even be evaluated when this feature is not present in image space.

After the equatorial streak has been associated to needleshaped domains, we try to analyze it quantitatively. In the complete scattering pattern $I\left(s_{12}, s_{3}\right)$ the isotropic feature and the streak are superimposed. Thus, the scattering of the streak

$$
I_{\mathrm{R}}\left(s_{12}, s_{3}\right)=I\left(s_{12}, s_{3}\right)-I_{i}(s)
$$

is separated from the isotropic scattering. From the length of the equatorial streak one may try to assess the diameter of the needle-shaped domains. For this purpose we have chosen a contour line at a height of $1 \%$ of the maximum intensity $I(0)$ of the pattern. This choice is rather arbitrary, because $I(0)$ is obtained by extrapolation into the blind area. Thus, the result is not only a function of the variation of the streak profile but also of the variation of $I(0)$. Moreover, if a contour at a different height level is chosen, the resulting value is shifted.

From the length $2 s_{12 n}$ of the area enclosed by the chosen contour, a needle diameter $d_{n}=1 / s_{12 n}$ is estimated. Figure 14 shows the result. As expected, stronger modulations are obtained after tomographic reconstruction. In contrast to this simple estimation, an absolute method for the analysis of equatorial streaks from needles has earlier been proposed (ref 27 and pp $166-170$ in ref 39$)$. For its application, the scattering $I_{\mathrm{R}}\left(s_{12}, s_{3}\right)$ of the streak is first projected

$$
\left\{I_{\mathrm{R}}\right\}_{2}\left(s_{12}\right)=\int I_{\mathrm{R}}\left(s_{12}, s_{3}\right) \mathrm{d} s_{3}
$$

on the cross section $s_{12}$ of the fiber. This curve can be analyzed like a scattering curve from the Kratky camera. It describes size and arrangement of the needle cross sections within the fiber cross section. The absolute method ${ }^{27}$ is significant, if roughness of the needle surfaces remains low and if the X-ray detector is not blind in the angular region where most of the needles are scattering. In this case the initial value, $g_{2}(0)$, of the $2 \mathrm{D}$ chord length distribution (CLD), $g_{2}\left(r_{12}\right)$, is lower than the maximum of the curve. This premise is never fulfilled for the data of this study. Figure 15 demonstrates the variation of the CLD as a function of the position on the fiber radius. We observe that 


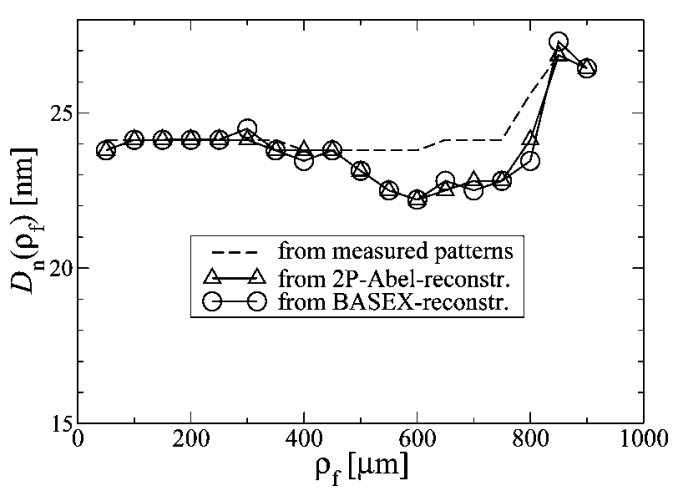

Figure 14. $\operatorname{MFC}\left(\lambda_{\mathrm{d}} \approx 3\right)$ in the scanning microbeam experiment. Spatial variation of a needle diameter, $d_{\mathrm{n}}\left(\rho_{\mathrm{f}}\right)$, as estimated from the length of the equatorial streak. $\rho_{\mathrm{f}}$ is the distance from the fiber axis. The dashed curve shows data determined from the measured data. The solid lines present the results obtained after tomographic reconstruction.

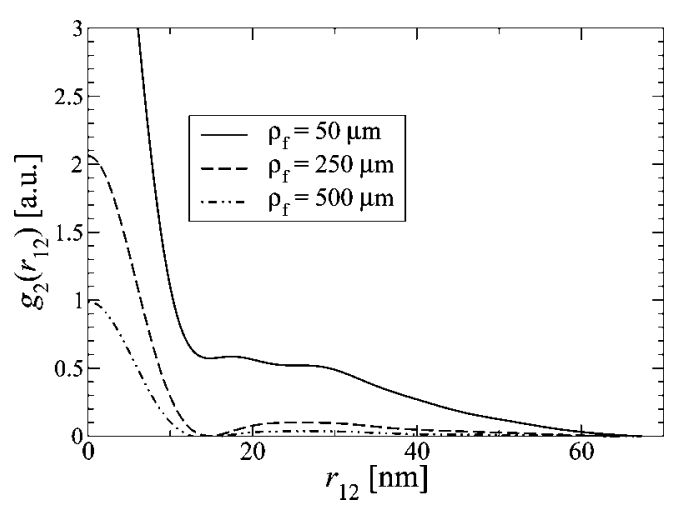

Figure 15. MFC $\left(\lambda_{d} \approx 3\right)$ in a scanning microbeam study. Analysis of the equatorial streaks from tomographically reconstructed scattering patterns. Chord length distribution (CLD) $g_{2}\left(r_{12}\right)$ of the cross sections of needle-shaped domains at various positions $\rho_{\mathrm{f}}$ along the fiber radius.

needle diameters above $60 \mathrm{~nm}$ are not resolved by our SAXS setup. The very strong roughness peak at small $r_{12}$ is found not only in the shown CLDs from the reconstructed patterns but also in the ones computed from the projected patterns. As $\rho_{\mathrm{f}}$ is increasing, the intensity of the CLD is strongly decreasing (Figure 15). Because the reconstructed patterns are normalized to constant voxel size, the analysis at least shows that in the center of the fiber the density of needle-shaped domains is much higher. Despite the severe problem with roughness and resolution limit, we extract ${ }^{27}$ the structure parameters from the CLD. Figure 16 shows the results. $A_{\mathrm{n}} / A_{\mathrm{V}}$ is a measure for the occupancy of the cross-sectional area of the voxel by needle cross sections. As already observed from the intensity of the CLD (Figure 15), the population density is rapidly decreasing away from the center of the fiber. The minimum is reached at $\rho_{\mathrm{f}}=600 \mu \mathrm{m}$. In the shell zone, slight increase of the needledomain population density is observed. Whereas in the discussion of the population density it appears acceptable to add-in the thin and rough chords, it appears bold in the description of both an average diameter, $\bar{D}_{\mathrm{n}}$, and of the width of the needle diameter distribution, $\sigma_{\mathrm{n}} / \bar{D}_{\mathrm{n}}$. Indeed, the computation of the parameters is mathematically correct, but for the physical nanostructure their discussion appears only meaningful if all needles are seen by the setup and the needle diameter distribution is (almost) vanishing at $r_{12}=0$. Thus, the different trends of $\bar{D}_{\mathrm{n}}$ and $D_{\mathrm{n}}$ (Figure 14) are attributed to the crude simplifications which cannot be avoided here.

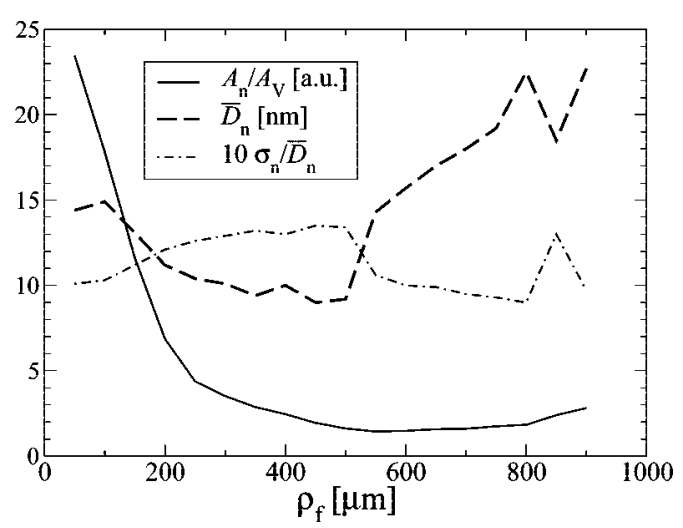

Figure 16. $\operatorname{MFC}\left(\lambda_{\mathrm{d}} \approx 3\right)$ in a scanning microbeam experiment. Evaluation of the equatorial streak scattering from reconstructed scattering patterns. Here, nanostructure parameters are extracted from the $2 \mathrm{D}$ chord length distribution $g_{2}\left(r_{12}\right)$ of the needle cross sections as a function of the position $\rho_{\mathrm{f}}$ along the fiber radius. $A_{\mathrm{n}} / A_{\mathrm{V}}$ is the total needle cross section per voxel cross section. $\bar{D}_{\mathrm{n}}$ number-average of the needle diameter distribution, and $\sigma_{\mathrm{n}} / \bar{D}_{\mathrm{n}}$ is the relative standard deviation of the needle diameter distribution.
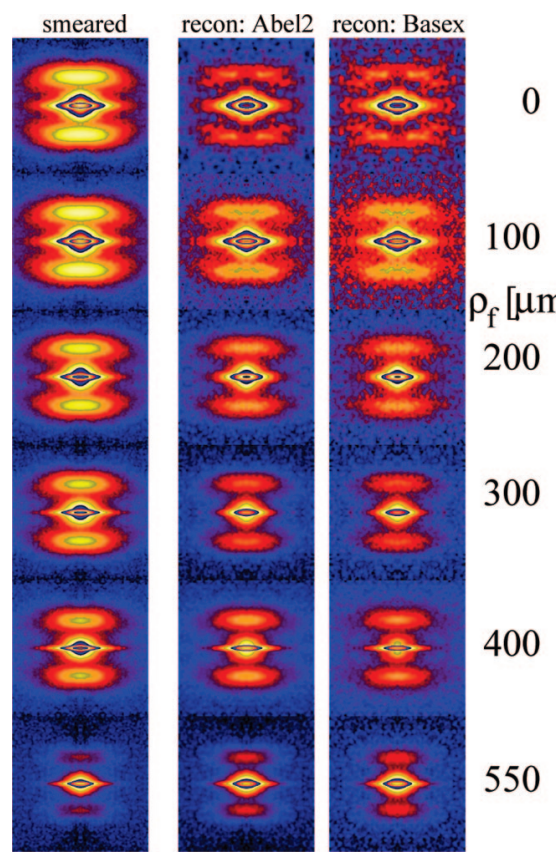

Figure 17. SAXS scattering patterns of $\operatorname{MFC}\left(\lambda_{d}=7\right)$. The fiber direction $s_{3}$ is vertical. The images present the range $-0.2 \mathrm{~nm}^{-1} \leq$ $s_{12}, s_{3} \leq 0.2 \mathrm{~nm}^{-1}$. The logarithmic intensity scaling is kept constant in each column. Left: measured SAXS patterns. Middle and right column: tomographically reconstructed patterns (two different methods) as a function of the distance $\rho_{\mathrm{f}}$ from the fiber axis.

4.6. $\mathrm{MFC} \lambda_{\mathbf{d}}=7$. Figure 17 presents the scattering patterns of the MFC which is cold-drawn to $\lambda_{\mathrm{d}} \approx 7$. Already the smeared recorded patterns exhibit that this material is much more homogeneous than the strand that is drawn to only $\lambda_{\mathrm{d}} \approx 3$. The reconstruction differences between both applied methods are small. All along the fiber radius, highly oriented layer line reflections of the PEBA material are found. Again, this feature is indicating highly oriented block stacks from alternating PEBA hard and soft domains which are arranged in fiber direction. The layer lines are even longer than those found with the 3-fold-drawn material. Thus, here the lateral extension of the hard-domain blocks is even narrower than in the 3-fold-drawn material. Close to the fiber axis $\left(0 \leq \rho_{\mathrm{f}} \leq 100 \mu \mathrm{m}\right)$ in the reconstructed patterns, we observe an indentation of the intensity at the meridian. This indicates arrangement of neighboring block stacks even in the 


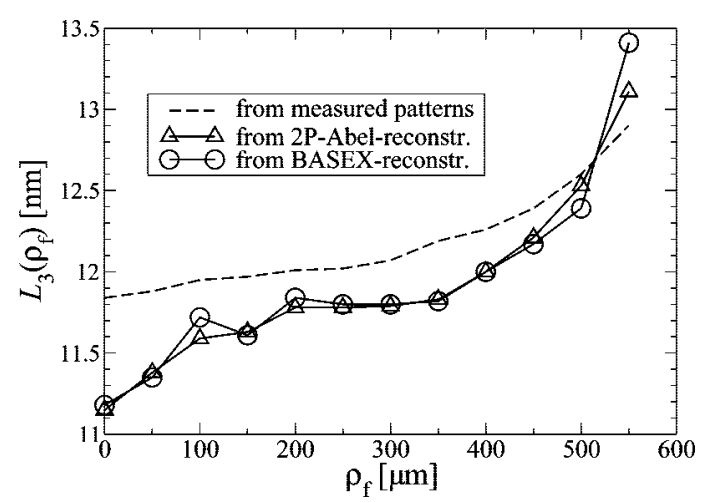

Figure 18. MFC $\left(\lambda_{d}=7\right)$ in a scanning microbeam experiment. Long period, $L_{3}\left(\rho_{\mathrm{f}}\right)$, as determined from the center of gravity of the layerline reflection, as a function of the distance $\rho_{\mathrm{f}}$ from the fiber axis. The dashed curve shows the result from projection space (measured data). Solid lines present the results from image space (i.e., after tomographic reconstruction by two different algorithms).

lateral direction. Further out, the distances between neighboring block stacks are random. With this highly drawn MFC, ultrareconstruction is very weak. It only results in a slight indentation close to the center of the scattering pattern, which has been eliminated by, again, cutting out a small circular region and extrapolation. In the central voxel we see little infrareconstruction. Thus, the conditions for perfect XSF-CT are almost fulfilled for this highly strained material: every local voxel emanates scattering that shows almost perfect fiber symmetry.

The position of the SAXS reflection on the meridian is almost constant. Nevertheless, because of the high orientation we can employ a determination method that permits to determine even small changes significantly. Instead of searching for the peak maximum, we cut out the layer-line peak along the contour through the saddle point toward the equatorial streak and determine its center of gravity, $s_{\mathrm{g}}=\left(s_{12 \mathrm{~g}}, s_{3 \mathrm{~g}}\right)=\left(0, s_{3 \mathrm{~g}}\right)$ by integration. Figure 18 presents the variation of the long period $L_{3}=1 / s_{3 \mathrm{~g}}$. The determination from the reconstructed patterns shows that $L_{3}$ of the PEBA blocks is lowest in the center of the fiber. After a slight increase it remains constant in the zones between $200 \mu \mathrm{m}$ and $350 \mu \mathrm{m}$, and increases again toward the fiber surface. As expected, this trend appears less pronounced when the smeared patterns are evaluated directly.

The existing saddle point between equatorial streak and layer line, as well, establishes a possibility to estimate a diameter of the PEBA blocks and of the PET microfibrils with less uncertainty than with the microfibrils of the 3-fold-drawn strand. For this purpose, we choose the lateral extensions of the contours through the saddle point.

The length $2 s_{12 \mathrm{~b}}$ of the region enclosed by the contour of the layer line reflection yields a relative measure of a block diameter $d_{\mathrm{b}}=1 / s_{12 \mathrm{~b}}$. Figure 19 demonstrates the result. The evaluation of the reconstructed pattern shows that the block diameters are following the trend of the long period between the blocks: low block diameters are correlated to low distances between them. Of course, also in this XSF-CT experiment a diameter of the PET needles can be extracted from the shape of the equatorial streak. The low significance resulting from needle roughness has already been demonstrated with the 3-fold-drawn MFC.

4.7. Visualization of Nanostructure before and after Tomographic Pattern Reconstruction. In an engineering polymer material, not only the diameters of needle-shaped domains exhibit broad distributions. In general, domain shape and arrangement are subjected to considerable fluctuation. One possibility to demonstrate the influence of these distributions on scattering data without making assumptions is the computation and visualization of the multidimensional chord distribution

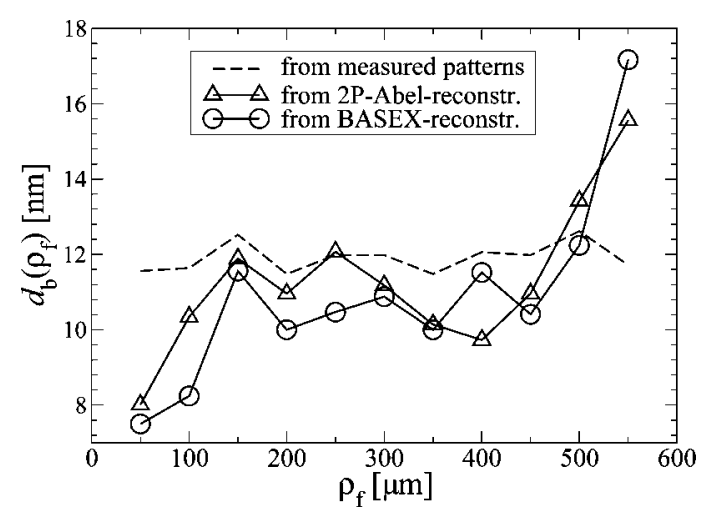

Figure 19. MFC $\left(\lambda_{\mathrm{d}} \approx 7\right)$ in the scanning microbeam experiment. Spatial variation of a block diameter, $d_{\mathrm{b}}\left(\rho_{\mathrm{f}}\right)$, as estimated from the length of the layer-line reflection. $\rho_{\mathrm{f}}$ is the distance from the fiber axis. The dashed curve shows data determined from the measured data. The solid lines present the results obtained after tomographic reconstruction.

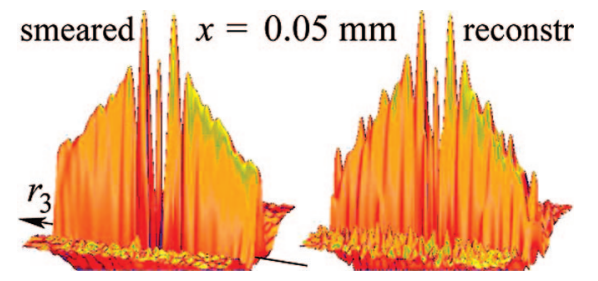

Figure 20. Demonstration of the desmearing effect by tomographic reconstruction on the nanostructure information as exhibited by the CDF $-z\left(r_{12}, r_{3}\right)$ (logarithmic scaling, $\left.-75 \mathrm{~nm} \leq r_{12}, r_{3} \leq 75 \mathrm{~nm}\right) . r_{3}$ is the direction of drawing. Nanostructure of the MFC $\left(\lambda_{\mathrm{d}}=7\right)$ at an transverse offset of $\rho_{\mathrm{f}}=50 \mu \mathrm{m}$ from the fiber axis.

(CDF). ${ }^{39,41}$ If, in a scanning-microbeam experiment, the measured pattern is integrating over many zones of a fiber with a structure gradient, the peaks in the CDF from the raw data must appear more blurred than those in a CDF computed from reconstructed patterns. Figure 20 shows a pair of corresponding CDFs. Obviously, the effect is strongest if the fiber is irradiated close to its axis where the raw pattern integrates over many zones without being disturbed from infrareconstruction.

We inspect the negative face $(-z(r))$ of the CDF, because the interesting distance distributions related to the block stacks are buried in a deep valley running along the meridian of the $\mathrm{CDF}$, which is generated by the PET microfibrils in the MFC material. Comparing in Figure 20 the projection space CDF with the image space CDF, the latter obviously shows narrower distance distribution peaks on the meridian $r_{3}$. A complete analysis of this structure requires a rather complex topological model that considers both components (needle scattering of the PET and the scattering from the PEBA block stacks).

Even though a quantitative analysis of this complex nanostructure appears to be too elaborate, at least the structure gradient along the fiber radius can be visualized and qualitatively described by the sequence of its CDFs (Figure 21). Only close to the center of the fiber the CDF shows several peaks that are not on the meridian. They describe the lateral correlation of hard domains that are forming the macrolattice, which has already been indicated in the corresponding scattering patterns (cf. Figure 17). Moving outward on the fiber radius, we observe up to $\rho_{\mathrm{f}}=100 \mu \mathrm{m}$ relatively broad domain peaks. Thus, close to the fiber axis, the block heights are subjected to considerable fluctuation. Further out, the block heights are rather uniform, but become broader again from $\rho_{\mathrm{f}}=350 \mu \mathrm{m}$. The region of narrow block height distributions more or less coincides with the range in Figure 18, in which the long period appears to be constant. Thus, we cannot exclude that a fraction of the observed 


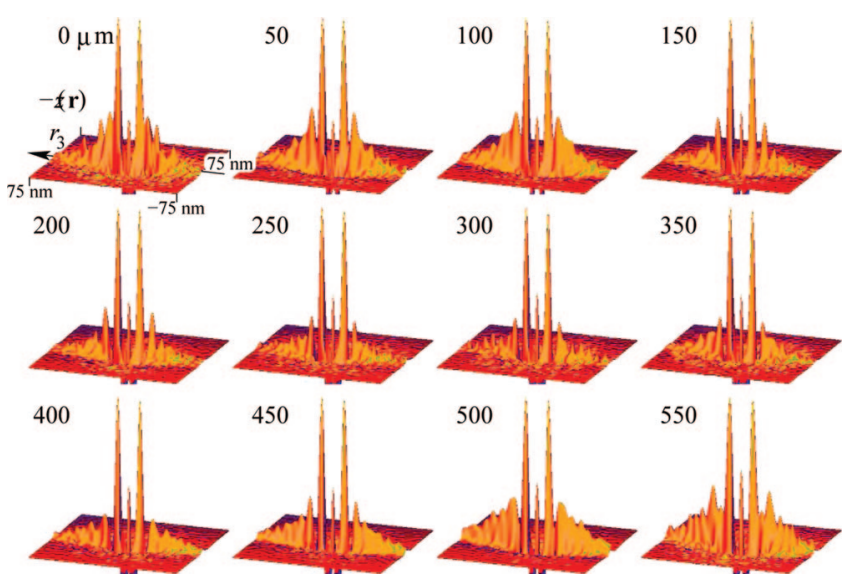

Figure 21. MFC cold drawn $\left(\lambda_{d}=7\right)$. Qualitative demonstration of the nanostructure gradient along the fiber radius from the fiber center $\left(\rho_{\mathrm{f}}=0 \mu \mathrm{m}\right)$ to the fiber surface $\left(\rho_{f}=550 \mu \mathrm{m}\right)$. The CDFs $-z\left(r_{12}, r_{3}\right)$ from the tomographically reconstructed scattering patterns are shown (linear scaling). $r_{3}$ is the direction of drawing.

broadening before and after this range is an artifact, which is generated from structure gradient within the local voxel (i.e., over a length of $40 \mu \mathrm{m}$ ). This shows that even in a tomographically reconstructed pattern from a scanning-microbeam experiment the effect of averaging over the volume irradiated by the microbeam should be borne in mind.

\section{Conclusions}

In the course of this investigation we have expanded our fundamental understanding of X-ray scattering fiber computer tomography. With this new ${ }^{21}$ method, a series of high-resolution $\mathrm{X}$-ray scattering patterns originating from scanning-microbeam experiments can be desmeared within a few minutes. Violation of local fiber symmetry not only affects the result of XSF-CT, but in similar manner the result of general SAXS tomography. Thus, we now are able to trace back the negative reconstructed intensities from an earlier general scattering tomography study $^{33,42}$ to the effect of ultrareconstruction that has been deduced here. We have demonstrated how to make use of ultrareconstruction. As a result, we now are able to discriminate between needle-shaped and shingle-shaped voids, if scattering data from a scanning-microbeam experiment are analyzed. In particular the as-extruded strand from neat PET exhibits many shingles, which are localized in the shell-zone of the strand. We have seen that the PET from our materials does not exhibit semicrystalline structure. Thus, it has only been possible to gather little information on the PET.

The neat PEBA always shows a long period of $10 \mathrm{~nm}$. Along the fiber radius and as a function of draw ratio, different orientations are observed. The as-extruded neat PEBA exhibits in its shell zone a remarkable long-period orientation on the equator, which is recovered as well in the composite. Even here, shingle voids are found in one of the outer zones. Further inward, the material appears isotropic. In the shell zones of the drawn PEBA, the scattering of block stacks oriented in fiber direction is found. The core of the strand does not show any scattering.

In both composite samples we find the PEBA scattering and an equatorial streak which is attributed to needle-shaped domains, because it shows LFS. We assign this effect to PET microfibrils. Not only in the neat PEBA but also in the composite that is drawn 3-fold (MFC3) there is a core in which the PEBA does not exhibit scattering. However, this zone is thinner in the MFC3 than in the PEBA. The breadth of the equatorial streak is varying along the fiber radius. Thus, the microfibrils are long in general, but close to the fiber axis and in the shell zone there are more but shorter microfibrils. The fibrils exhibit considerable roughness. Thus, the significance of a shape analysis is low. We suppose that we have only caught a small fraction of the needles in our SAXS experiment. This will possibly change, as we proceed from microfibrillarreinforced composites (MFC) to the study of nanofibrillarreinforced composites (NFC).

The composite that is drawn 7-fold (MFC7) exhibits a much more homogeneous structure than the MFC3. Along the complete fiber radius a layer-line pattern with the PEBA's long period is observed. Close to the axis of the MFC7 strand an indication of lateral correlation between the block stacks of the PEBA is observed. Such an effect has frequently been found after a drawing of polymer materials. ${ }^{4-49}$ MFC7 is very close to local fiber symmetry. Thus, application of XSF-CT results in almost perfect reconstruction of the nanostructure.

Considering possible advanced studies, it would be of academic interest to monitor the progress of the oriented PEBA structure toward the core of the strand. Is there a phase interface moving inward, or does the PEBA orientation gradually change in the volume? Anticipating possible relaxation effects in the elastic PEBA, it could be promising to carry out in situ XSFCT during ${ }^{50}$ slow cold-drawing. Utilizing a dedicated microbeam station, such experiments could eventually be carried out with a time resolution of $2 \mathrm{~min}$. In general, our rather coarse spatial resolution of $50 \mu \mathrm{m}$ has proved sufficient for the study of these materials. Nevertheless, some minor questions that have already been mentioned in the discussion (narrow shingle zone; structure gradient within the voxel) can be answered by application of a finer microbeam. On the other hand, the study of structure gradients in polymer parts (e.g., pipes, rods) does not require a fine microbeam. Such parts may be specially prepared for the reason to study fundamental questions, or the precondition of fiber symmetry can be checked with little effort.

Acknowledgment. We acknowledge HASYLAB, Hamburg, for provision of the synchrotron radiation facilities at beamline BW4 in the frame of project II-04-039. U.N. thanks Wellstream Inc., Newcastle, GB, for the opportunity to carry out a survey on damaged pipelines for his living.

\section{References and Notes}

(1) Evstatiev, M.; Fakirov, S. Polymer 1992, 33, 877.

(2) Evstatiev, M.; Nicolov, N.; Fakirov, S. Polymer 1996, 37, 4455.

(3) Fakirov, S.; Stribeck, N.; Apostolov, A. A.; Denchev, Z.; Krasteva, B.; Evstatiev, M.; Friedrich, K. J. Macromol. Sci. Phys. 2001, 40, 935.

(4) Schmidt, P.; Baldrian, J.; Ščudla, J.; Dybal, J.; Raab, M.; Eichhorn, K.-J. Polymer 2001, 41, 5321.

(5) Li, Z.-M.; Yang, M.-B.; Feng, J.-M.; Yang, W.; Huang, R. Mater. Res. Bull. 2002, 37, 2185.

(6) Kajiura, Y.; Watanabe, S.; Itou, T.; Iida, A.; Shinohara, Y.; Amemiya, Y. J. Appl. Crystallogr. 2005, 38, 420.

(7) Riekel, C.; Engstrom, P.; Martin, C. J. Macromol. Sci., Phys. 1998, B37, 587 .

(8) Riekel, C. Rep. Prog. Phys. 2000, 63, 233.

(9) Riekel, C.; García Gutiérrez, M. C.; Gourrier, A.; Roth, S. V. Anal. Bioanal. Chem 2003, 376, 594.

(10) Nozue, Y.; Shinohara, Y.; Amemiya, Y. Polymer J. 2007, 39, 1221.

(11) Müller, M.; Czihak, C.; Vogl, G.; Fratzl, P.; Schober, H.; Riekel, C. Macromolecules 1998, 31, 3953.

(12) Wang, Y. D.; Cakmak, M. Polymer 2001, 42, 4233.

(13) Paris, O.; Loidl, D.; Müller, M.; Lichtenegger, H.; Peterlik, H. J. Appl. Crystallogr. 2001, 34, 473.

(14) Loidl, D.; Peterlik, H.; Paris, O.; Müller, M.; Burghammer, M.; Riekel, C. J. Synchrotron Radiat. 2005, 12, 758.

(15) Paris, O.; Loidl, D.; Peterlik, H.; Müller, M.; Lichtenegger, H.; Fratzl, P. J. Appl. Crystallogr. 2000, 33, 695.

(16) Paris, O.; Loidl, D.; Peterlik, H. Carbon 2002, 40, 551.

(17) Müller, M.; Czihak, C.; Burghammer, M.; Riekel, C. J. Appl. Crystallogr. 2000, 33, 817.

(18) Müller, M.; Riekel, C.; Vuong, R.; Chanzy, H. Polymer 2000, 41, 2627. 
(19) Lozano-Castelló, D.; Raymundo-Pinero, E.; Cazorla-Amorós, D.; Linares-Solano, A.; Müller, M.; Riekel, C. Carbon 2002, 40, 2727.

(20) Flores, A.; Poeppel, A.; Riekel, C.; Schulte, K. J. Macromol. Sci. Phys. 2001, 40, 749.

(21) Stribeck, N.; Nöchel, U.; Almendárez Camarillo, A. Macromol. Chem. Phys. 2008, in press (DOI: 10.1002/macp.200800242).

(22) Dasch, C. J. Appl. Opt. 1992, 31, 1146.

(23) Dribinsi, V.; Ossadtchi, A.; Mandelshtam, V. A.; Reisler, H. Rev. Sci. Instrum. 2002, 73, 2634.

(24) Davies, R. J.; Koenig, C.; Burghammer, M.; Riekel, C. Appl. Phys. Lett. 2008, 92, 101903.

(25) Bonart, R. Kolloid Z. Z. Polym. 1966, 211, 14.

(26) Stribeck, N. ACS Symp. Ser. 2000, 739, 41.

(27) Stribeck, N. J. Polym. Sci., Part B: Polym. Phys. 1999, 37, 975.

(28) Peterlin, A. Text. Res J. 1972, 42, 20.

(29) Bitter, I.; Kaufman, A. E.; Sato, M. IEEE Trans. Visualization Comput. Graphics 2001, 7, 195.

(30) Abel, N. H. J. Reine Angew. Math. 1826, 1, 153.

(31) Guinier, A.; Fournet, G. Nature 1947, 160, 501.

(32) DuMond, J. W. M. Phys. Rev. 1947, 72, 83.

(33) Stribeck, N.; Almendarez Camarillo, A.; Nöchel, U.; Schroer, C.; Kuhlmann, M.; Roth, S. V.; Gehrke, R.; Bayer, R. K. Macromol. Chem. Phys. 2006, 207, 1239.

(34) Gull, S. F.; Skilling, J. IEE Proc. Part F 1984, 131, 646.

(35) Haykin, S., Ed.; Blind deconvolution; Prentice Hall PTR: Englewood Cliffs, NJ, 1994.

(36) Stribeck, N.; Nöchel, U. J. Appl. Crystallogr. 2008, 41, 715.
(37) Lengeler, B.; Tümmler, J.; Snigirev, A.; Snigireva, I.; Raven, C. J. Appl. Phys. 1998, 84, 5855.

(38) Schroer, C. G.; Kuhlmann, M.; Lengeler, B.; Günzler, T. F.; Kurapova, O.; Benner, B.; Rau, C.; Simionovici, A. S.; Snigirev, A. A.; Snigireva, I. Proc. SPIE 2002, 4783, 10.

(39) Stribeck, N. X-Ray Scattering of Soft Matter; Springer: Heidelberg, Germany, 2007.

(40) Fronk, W.; Wilke, W. Colloid Polym. Sci. 1985, 263, 97.

(41) Stribeck, N. J. Appl. Crystallogr. 2001, 34, 496.

(42) Schroer, C. G.; Kuhlmann, M.; Roth, S. V.; Gehrke, R.; Stribeck, N.; Almendarez Camarillo, A.; Lengeler, B. Appl. Phys. Lett. 2006, 88, 164102 .

(43) Hosemann, R.; Wilke, W. Faserforsch. Textiltech. 1964, 15, 521.

(44) Campos Lopez, E.; McIntyre, D.; Fetters, L. J. Macromolecules 1973, 6,415 .

(45) Matyi, R. J.; Crist, B., Jr. J. Polym. Sci. Polym. Phys. 1978, 16, 1329.

(46) Wilke, W.; Bratrich, M. J. Appl. Crystallogr. 1991, 24, 645.

(47) Stribeck, N.; Sapoundjieva, D.; Denchev, Z.; Apostolov, A. A.; Zachmann, H. G.; Stamm, M.; Fakirov, S. Macromolecules 1997, 30, 1329.

(48) Stribeck, N.; Androsch, R.; Funari, S. S. Macromol. Chem. Phys. 2003, 204, 1202.

(49) Stribeck, N. In Condensation Thermoplastic Elastomers; Fakirov, S., Ed.; Wiley-VCH: Weinheim, Germany, 2005; pp 197-225.

(50) Stribeck, N.; Nöchel, U.; Funari, S. S.; Schubert, T. J. Polym. Sci. Polym. Phys. 2008, 46, 721.

MA8015322 